\title{
A New Type of Coincidence and Common Fixed Point Theorem with Applications
}

\author{
Shyam Lal Singh, ${ }^{1}$ Raj Kamal, ${ }^{2}$ Manuel De la Sen, ${ }^{3}$ and Renu Chugh ${ }^{2}$ \\ ${ }^{1}$ Gurukula K. Vishwavidyalaya, Haridwar 249404, India \\ ${ }^{2}$ Department of Mathematics, Maharshi Dayanand University, Rohtak 124001, India \\ ${ }^{3}$ Institute of Research and Development Processes, University of Basque Country, Campus of Leioa (Bizkaia), \\ Apartado 644, 48080 Bilbao, Spain
}

Correspondence should be addressed to Shyam Lal Singh; vedicmri@gmail.com

Received 26 September 2013; Accepted 24 March 2014; Published 30 April 2014

Academic Editor: Douglas R. Anderson

Copyright (C) 2014 Shyam Lal Singh et al. This is an open access article distributed under the Creative Commons Attribution License, which permits unrestricted use, distribution, and reproduction in any medium, provided the original work is properly cited.

Coincidence and common fixed point theorems for a class of Ćirić-Suzuki hybrid contractions involving a multivalued and two single-valued maps in a metric space are obtained. Some applications including the existence of a common solution for certain class of functional equations arising in a dynamic programming are also discussed.

\section{Introduction}

Consistent with [1] (see also [2, 3]), $Y$ denotes an arbitrary nonempty set, $(X, d)$ a metric space, and $C L(X)$ (resp., $C B(X)$ ), the collection of all nonempty closed (resp., closed bounded) subsets of $X$. The hyperspace $(C L(X), H)$ (resp., $(C B(X), H))$ is called the generalized Hausdorff (resp., the Hausdorff) metric space induced by the metric $d$ on $X$.

For nonempty subsets $A, B$ of $X, d(A, B)$ denotes the gap between the subsets $A$ and $B$, while

$\rho(A, B)=\sup \{d(a, b): a \in A, b \in B\}$,

$B N(X)=\{A: \emptyset \neq A \subseteq X$ and the diameter of $A$ is finite $\}$.

As usual, we write $d(x, B)$ (resp., $\rho(x, B)$ ) for $d(A, B)$ (resp., $\rho(A, B))$ when $A=\{x\}$.

For the sake of brevity, we follow the following notations, wherein $S, f$, and $g$ are maps to be defined specifically in a particular context, while $x$ and $y$ are elements of some specific domain:

$$
\begin{aligned}
& M(S ; f x, g y) \\
& =\max \left\{\begin{array}{l}
d(f x, g y), d(f x, S x), d(g y, S y), \\
\left.\frac{d(S x, g y)+d(S y, f x)}{2}\right\} ;
\end{array}\right. \\
& M(S ; f x, f y) \\
& =\max \{d(f x, f y), d(f x, S x), d(f y, S y), \\
& \left.\frac{d(S x, f y)+d(S y, f x)}{2}\right\} ;
\end{aligned}
$$

$$
\begin{gathered}
M(S x, S y) \\
=\max \{d(x, y), d(x, S x), d(y, S y), \\
\left.\frac{d(y, S x)+d(x, S y)}{2}\right\} ;
\end{gathered}
$$




$$
\begin{gathered}
M_{1}(f x, g y) \\
=\max \{d(x, y), d(x, f x), d(y, g y), \\
\left.\frac{d(y, f x)+d(x, g y)}{2}\right\} .
\end{gathered}
$$

The Banach contraction principle $(\mathrm{Bcp})$ plays an important role in nonlinear analysis and has numerous generalizations and several applications (see, e.g., [1-21] and others). Nadler Jr. [1] (see also [22]) initiated the study of multivalued Banach contractions in metric spaces. In view of its numerous applications, the Nadler multivalued contraction theorem received enormous attention (see, e.g., $[2,3,7,8,11-15,17-$ 21, 23-36] and references thereof).

The following result $[13$, p. 250$]$ extends and generalizes many results due to Fisher [37], Goebel [38], Kubiak [29], and others.

Theorem 1. Let $S: Y \rightarrow C L(X)$ and $f, g: Y \rightarrow X$ be such that $S(Y) \subseteq f(Y) \cap g(Y)$, and one of $S(Y), f(Y)$ or $g(Y)$ is a complete subspace of $X$. Assume there exists $r \in[0,1)$ such that for every $x, y \in Y$,

$$
H(S x, S y) \leq r M(S ; f x, g y)
$$

Then

(i) $S$ and $f$ have a coincidence point $v$ in $Y$,

(ii) $S$ and $g$ have a coincidence point $w$ in $Y$. Further, if $Y=X$, then

(iii) $S$ and $f$ have a common fixed point $v$ provided that $f v$ is a fixed point of $f$, and $f$ and $S$ commute at $v$;

(iv) $S$ and $g$ have a common fixed point $w$ provided that gw is a fixed point of $g$, and $g$ and $S$ commute at $w$;

(v) $S, f$, and $g$ have a common fixed point provided that (iii) and (iv) both are true.

We remark that certain contractive conditions studied for $S: Y \rightarrow C L(X)$ and $f, g: Y \rightarrow X$ by Ćirić [5], Covitz and Nadler Jr. [16], Czerwik [6], Fisher [37], Goebel [38], Jungck [17], Kubiak [29], Naimpally et al. [8], Pathak [15], Pathak et al. [9], Petrusel and Rus [10], Reich [11], and Rus [3] are included in the following condition:

$$
H(S x, S y) \leq r M(S ; f x, g y)
$$

for every $x, y \in Y$, where $0 \leq r<1$.

In particular, (4) with $Y=X$ and $f=g=$ the identity map on $X$ was studied by Ćirić [5].

Recently, Suzuki [39, Th. 2] obtained a remarkable generalization of the Bcp. The same has been extended to multivalued maps by Kikkawa and Suzuki [30] in the following manner.
Theorem 2. Define a strictly decreasing function $\eta:[0,1) \rightarrow$ $((1 / 2), 1]$ by

$$
\eta(r)=\frac{1}{1+r}
$$

Let $(X, d)$ be a complete metric space and $S: Y \rightarrow C B(X)$. Assume there exists $r \in[0,1)$ such that for every $x, y \in Y$,

$$
\eta(r) d(x, S x) \leq d(x, y) \quad \text { implies } H(S x, S y) \leq r d(x, y) .
$$

Then there exists $z \in X$ such that $z \in S z$.

Subsequently, some interesting extensions and generalizations of Theorem 2 were obtained among others by Abbas et al. [23], Dhompongsa and Yingtaweesittikul [24], Dorić and Lazović [25], Kamal et al. [18], Moț and Petruşel [26], Singh and Mishra [27, 31, 36], and Singh et al. [28, 32, 33].

The importance of Suzuki contraction theorem [39, Th. 2] and subsequently obtained coincidence and fixed point theorems (cf. [23-28, 30-33, 36] and others) for maps in metric spaces satisfying Suzuki-type contractive conditions is that the contractive conditions are required to be satisfied not for all points of the domain.

In all that follows we take a nonincreasing function $\varphi$ from $[0,1)$ onto $(0,1]$ defined by

$$
\varphi(r)= \begin{cases}1 & \text { if } 0 \leq r<\frac{1}{2} \\ 1-r & \text { if } \frac{1}{2} \leq r<1 .\end{cases}
$$

Recently, Singh et al. [33] obtained the following coincidence and common fixed point theorem which is a generalization of a result of Dorić and Lazović [25].

Theorem 3. Let $S: Y \rightarrow C L(X)$ and $f: Y \rightarrow X$ be such that $S(Y) \subseteq f(Y)$. Assume there exists $r \in[0,1)$ such that for every $x, y \in Y$,

$$
\begin{array}{r}
\varphi(r) d(f x, S x) \leq d(f x, f y) \\
\text { implies } H(S x, S y) \leq r M(S ; f x, f y) .
\end{array}
$$

If one of $S(Y)$ or $f(Y)$ is a complete subspace of $X$, then there exists a point $z \in Y$ such that $f z \in S z$.

Further, if $Y=X$ and $f z$ is a fixed point of $f$, then $f z$ is a fixed point of $S$ provided that $f$ is IT-commuting with $S$ at $z$.

In this paper, we obtain a coincidence and common fixed point theorem (cf. Theorem 6) extending and generalizing Theorems 1, 2, 3, and several others. We also deduce the existence of common solution for a certain class of functional equations arising in dynamic programming. Examples are given to justify theorems and applications.

\section{Main Results}

The following definition is due to Itoh and Takahashi [19] (see also [27]). 
Definition 4. Let $S: X \rightarrow C L(X)$ and $f: X \rightarrow X$. Then the hybrid pair $(S, f)$ is IT-commuting at $z \in X$ if $f S z \subseteq S f z$.

We remark that IT-commuting maps are more general than commuting maps $[34$, p. 2]. However, a pair of maps $f, g: X \rightarrow X$ are IT-commuting (also called weakly compatible by Jungck and Rhoades [20]) at $x \in X$ if $f g x=$ $g f x$ when $f x=g x$.

We will need the following lemma essentially due to Nadler Jr. [1] (see also [5], [2, p. 61], [35, p. 4], [3, p. 76]).

Lemma 5. If $A, B \in C L(X)$ and $a \in A$, then for each $\varepsilon>0$, there exists $b \in B$ such that $d(a, b) \leq H(A, B)+\varepsilon$.

Let $C(S, f)$ denote the collection of all coincidence points of $S$ and $f$; that is, $C(S, f)=\{z \in Y: f z \in S z\}$ when $S: Y \rightarrow$ $C L(X)$ and $f: Y \rightarrow X$; and $C(S, f)=\{z \in Y: f z=S z\}$ when $S, f: Y \rightarrow X$.

The following is the main result of this section.

Theorem 6. Let $S: Y \rightarrow C L(X)$ and $f, g: Y \rightarrow X$ be such that $S(Y) \subseteq f(Y) \cap g(Y)$. Assume there exists $r \in[0,1)$ such that for every $x, y \in Y$,

$$
\varphi(r) \min \{d(f x, S x), d(g y, S y)\} \leq d(f x, g y)
$$

implies

$$
H(S x, S y) \leq r M(S ; f x, g y) .
$$

If one of $S(Y), f(Y)$, or $g(Y)$ is a complete subspace of $X$, then

(I) $C(S, f)$ is nonempty; that is, there exists a point $z \in Y$ such that $f z \in S z$.

(II) $C(S, g)$ is nonempty; that is, there exists a point $z_{1} \in Y$ such that $g z_{1} \in S z_{1}$.

Further if, $Y=X$, then

(III) $S$ and $f$ have a common fixed point provided that the maps $S$ and $f$ are IT-commuting just at coincidence point $z$ and $f z$ is fixed point of $f$;

(IV) $S$ and $g$ have a common fixed point provided that the maps $S$ and $g$ are IT-commuting just at coincidence point $z_{1}$ and $g z_{1}$ is fixed point of $g$;

(V) $S, f$, and $g$ have a common fixed point provided that both (III) and (IV) are true.

Proof. Without loss of generality, we may take $r>0$ and $f, g$ nonconstant maps.

Let $\varepsilon>0$ be such that $\beta=r+\varepsilon<1$. We construct two sequences $\left\{x_{n}\right\}$ in $Y$ and $\left\{y_{n}\right\}$ in $X$ as follows.

Let $x_{0} \in Y$ and $y_{0}=g x_{1} \in S x_{0}$. By Lemma 5, there exists $y_{1}=f x_{2} \in S x_{1}$ such that

$$
d\left(f x_{2}, g x_{1}\right) \leq H\left(S x_{0}, S x_{1}\right)+\varepsilon M\left(S ; f x_{0}, g x_{1}\right) .
$$

Similarly, there exists $y_{2}=g x_{3} \in S x_{2}$ such that

$$
d\left(f x_{2}, g x_{3}\right) \leq H\left(S x_{2}, S x_{1}\right)+\varepsilon M\left(S ; f x_{2}, g x_{1}\right) .
$$

Continuing in this manner, we find a sequence $\left\{y_{n}\right\}$ in $X$ such that

$$
\begin{aligned}
& y_{2 n}=g x_{2 n+1} \in S x_{2 n}, \quad y_{2 n+1}=f x_{2 n+2} \in S x_{2 n+1}, \\
& d\left(f x_{2 n}, g x_{2 n+1}\right) \\
& \quad \leq H\left(S x_{2 n}, S x_{2 n-1}\right)+\varepsilon M\left(S ; f x_{2 n}, g x_{2 n-1}\right) \\
& d\left(f x_{2 n+2}, g x_{2 n+1}\right) \\
& \quad \leq H\left(S x_{2 n}, S x_{2 n+1}\right)+\varepsilon M\left(S ; f x_{2 n}, g x_{2 n+1}\right) .
\end{aligned}
$$

Now, we show that for any $n \in N$,

$$
d\left(y_{2 n}, y_{2 n-1}\right) \leq \beta d\left(y_{2 n-1}, y_{2 n-2}\right) \text {. }
$$

Suppose if $d\left(g x_{2 n-1}, S x_{2 n-1}\right) \geq d\left(f x_{2 n}, S x_{2 n}\right)$, then

$$
\begin{aligned}
& \varphi(r) \min \left\{d\left(f x_{2 n}, S x_{2 n}\right), d\left(g x_{2 n-1}, S x_{2 n-1}\right)\right\} \\
& \leq d\left(f x_{2 n}, g x_{2 n-1}\right) .
\end{aligned}
$$

Therefore, by the assumption,

$$
\left.\begin{array}{l}
d\left(f x_{2 n}, g x_{2 n+1}\right) \\
\quad \leq H\left(S x_{2 n}, S x_{2 n-1}\right)+\varepsilon M\left(S ; f x_{2 n}, g x_{2 n-1}\right) \\
\leq r M\left(S ; f x_{2 n}, g x_{2 n-1}\right)+\varepsilon M\left(S ; f x_{2 n}, g x_{2 n-1}\right) \\
=\beta M\left(S ; f x_{2 n}, g x_{2 n-1}\right) \\
=\beta \max \left\{d\left(f x_{2 n}, g x_{2 n-1}\right), d\left(f x_{2 n}, S x_{2 n}\right),\right. \\
\quad \frac{d\left(g x_{2 n-1}, S x_{2 n-1}\right),}{d\left(g x_{2 n-1}, S x_{2 n}\right)+d\left(f x_{2 n}, S x_{2 n-1}\right)}
\end{array}\right\} .
$$

This yields (13).

Suppose if $d\left(f x_{2 n}, S x_{2 n}\right) \geq d\left(g x_{2 n-1}, S x_{2 n-1}\right)$, then

$$
\begin{aligned}
& \varphi(r) \min \left\{d\left(f x_{2 n}, S x_{2 n}\right), d\left(g x_{2 n-1}, S x_{2 n-1}\right)\right\} \\
& \leq d\left(f x_{2 n}, g x_{2 n-1}\right) .
\end{aligned}
$$


Therefore, by the assumption,

$$
\begin{aligned}
d\left(f x_{2 n}, g x_{2 n+1}\right) & \\
\leq & H\left(S x_{2 n}, S x_{2 n-1}\right)+\varepsilon M\left(S ; f x_{2 n}, g x_{2 n-1}\right) \\
& \leq r M\left(S ; f x_{2 n}, g x_{2 n-1}\right)+\varepsilon M\left(S ; f x_{2 n}, g x_{2 n-1}\right) \\
& =\beta M\left(S ; f x_{2 n}, g x_{2 n-1}\right) \\
& =\beta \max \left\{d\left(f x_{2 n}, g x_{2 n-1}\right), d\left(f x_{2 n}, S x_{2 n}\right),\right. \\
& \left.\quad \frac{d\left(g x_{2 n-1}, S x_{2 n-1}\right),}{2}, S x_{2 n-1}\right)+d\left(f x_{2 n}, S x_{2 n-1}\right) \\
\leq & \beta \max \left\{d\left(f x_{2 n}, g x_{2 n-1}\right), d\left(f x_{2 n}, g x_{2 n+1}\right)\right\}
\end{aligned}
$$

yielding (13). So, in both cases, we obtain (13). In an analogous manner, we show that

$$
d\left(y_{2 n+1}, y_{2 n}\right) \leq \beta d\left(y_{2 n}, y_{2 n-1}\right) .
$$

We conclude from (13) and (18) that for any $n \in N$,

$$
d\left(y_{n+1}, y_{n}\right) \leq \beta d\left(y_{n}, y_{n-1}\right) \text {. }
$$

Therefore the sequence $\left\{y_{n}\right\}$ is Cauchy. Assume that the space $g(Y)$ is complete. Notice that the sequence $\left\{y_{2 n}\right\}$ is contained in $g(Y)$ and has a limit in $g(Y)$. Call it $u$. Let $z \in f^{-1} u$. Then $z \in Y$ and $f z=u$. The subsequence $\left\{y_{2 n+1}\right\}$ also converges to $u$. Let $z_{1} \in g^{-1} u$. Then

$$
g z_{1}=u
$$

Now we show that for any $g y \in X-\{f z\}$,

$$
d(u, S y) \leq r \max \{d(u, g y), d(g y, S y)\},
$$

and for any $f y \in X-\{g z\}$,

$$
d(u, S y) \leq r \max \{d(u, f y), d(f y, S y)\} .
$$

Since $f x_{2 n} \rightarrow f z$, there exists $n_{0} \in N$ (naturals) such that

$$
d\left(f x_{2 n}, f z\right) \leq \frac{1}{3} d(f z, g y) \quad \text { for } g y \neq f z \text { and all } n \geq n_{0} .
$$

Also, since $g x_{2 n+1} \rightarrow f z$, there exists $n_{1} \in N$ such that

$$
\begin{gathered}
d\left(g x_{2 n+1}, f z\right) \leq \frac{1}{3} d(f z, g y) \\
\text { for } g y \neq f z \text { and all } n \geq n_{1} .
\end{gathered}
$$

Then, as in [39, p. 1862] (see also [25]),

$$
\begin{aligned}
\varphi(r) & d\left(f x_{2 n}, S x_{2 n}\right) \\
& \leq d\left(f x_{2 n}, S x_{2 n}\right) \leq d\left(f x_{2 n}, g x_{2 n+1}\right) \\
& \leq \frac{2}{3} d(f z, g y)=d(f z, g y)-\frac{1}{3} d(f z, g y) \\
& \leq d(f z, g y)-d\left(f x_{2 n}, f z\right) \leq d\left(f x_{2 n}, g y\right) .
\end{aligned}
$$

Therefore,

$$
\varphi(r) d\left(f x_{2 n}, S x_{2 n}\right) \leq d\left(f x_{2 n}, g y\right)
$$

Now, either $d\left(f x_{2 n}, S x_{2 n}\right) \leq d(g y, S y)$ or $d(g y, S y) \leq$ $d\left(f x_{2 n}, S x_{2 n}\right)$.

In each case, by (26) and the assumption,

$$
\begin{gathered}
d\left(f x_{2 n+1}, S y\right) \\
\leq H\left(S x_{2 n}, S y\right) \leq r M\left(S ; f x_{2 n}, g y\right) . \\
\leq r \max \left\{d\left(f x_{2 n}, g y\right), d\left(f x_{2 n}, S x_{2 n}\right), d(g y, S y),\right. \\
\left.\frac{d\left(f x_{2 n}, S y\right)+d\left(g y, S x_{2 n}\right)}{2}\right\} .
\end{gathered}
$$

Making $n \rightarrow \infty$,

$$
\begin{aligned}
& d(u, S y) \\
& \leq r \max \{d(u, g y), d(u, u), d(g y, S y), \\
& \left.\quad \frac{d(u, S y)+d(u, g y)}{2}\right\} \\
& \leq r \max \left\{d(u, g y), d(g y, S y), \frac{d(u, S y)+d(u, g y)}{2}\right\} \\
& =r \max \{d(u, g y), d(g y, S y)\} .
\end{aligned}
$$

This yields (21); that is,

$$
d(f z, S y) \leq r \max \{d(f z, g y), d(g y, S y)\}
$$

Analogously, we can prove (22); that is,

$$
d\left(g z_{1}, S y\right) \leq r \max \left\{d\left(g z_{1}, f y\right), d(f y, S y)\right\} .
$$

Now, we show that $C(S, f)$ is nonempty.

We first consider the case $0 \leq r<1 / 2$.

Suppose $f z \notin S z$. Then as in [24, p. 6], let $g a \in S z$ be such that $2 r d(g a, f z)<d(S z, f z)$.

Since $g a \in S z$ implies $g a \neq f z$, we have from (21) and (22),

$$
d(f z, S a) \leq r \max \{d(f z, g a), d(g a, S a)\}
$$

On the other hand, since $\varphi(r) d(f z, S z) \leq d(f z, S z) \leq$ $d(f z, g a)$,

$$
\varphi(r) \min \{d(f z, S z), d(g a, S a)\} \leq d(f z, g a) .
$$


Therefore, by the assumption (13),

$$
\begin{aligned}
d(g a, S a) & \leq H(S z, S a) \\
& \leq r \max \{d(f z, g a), d(f z, S z), d(g a, S a), \\
\left.\frac{d(f z, S a)+d(g a, S z)}{2}\right\} & r \max \{d(f z, g a), d(g a, S a)\} .
\end{aligned}
$$

This gives $d(g a, S a) \leq H(S z, S a) \leq r d(f z, g a)<d(f z, g a)$. tion,

So by $(31), d(f z, S a) \leq r d(f z, g a)$. Thus, by the assump-

$$
\begin{aligned}
d(f z, S z) & \leq d(f z, S a)+H(S z, S a) \\
& \leq r d(f z, g a)+r d(f z, g a) \\
& =2 r d(f z, g a)<d(f z, S z) .
\end{aligned}
$$

This contradicts $f z \notin S z$. Consequently, $f z \in S z$, and $C(S, f)$ is nonempty.

In an analogous manner, we can prove in the case $0 \leq r<$ $1 / 2$ that $C(S, g)$ is nonempty.

We now consider the case $1 / 2 \leq r<1$. We first show that

$$
\begin{aligned}
& H(S z, S y) \\
& \leq r \max \{d(f z, g y), d(f z, S z), d(g y, S y), \\
& \left.\frac{d(g y, S z)+d(f z, S y)}{2}\right\} .
\end{aligned}
$$

Assume that $f z \neq g y$. Then for every $n \in N$, there exists $z_{n} \in$ Sy such that

$$
d\left(f z, z_{n}\right) \leq d(f z, S y)+\frac{1}{n} d(f z, g y) .
$$

Therefore,

$$
\begin{aligned}
d(g y, S y) & \leq d\left(g y, z_{n}\right) \\
& \leq d(g y, f z)+d\left(f z, z_{n}\right) \\
& \leq d(g y, f z)+d(f z, s y)+\frac{1}{n} d(f z, g y) .
\end{aligned}
$$

So using (31), the inequality (37) implies

$$
\begin{aligned}
d(g y, S y) \leq & d(f z, g y)+r \max \{d(f z, g y), d(g y, S y)\} \\
& +\frac{1}{n} d(f z, g y) .
\end{aligned}
$$

If $d(f z, g y) \geq d(g y, S y)$, then (38) gives

$$
\begin{aligned}
d(g y, S y) & \leq d(f z, g y)+r d(f z, g y)+\frac{1}{n} d(f z, g y) \\
& =\left(1+r+\frac{1}{n}\right) d(f z, g y) .
\end{aligned}
$$

Making $n \rightarrow \infty$,

$$
d(g y, S y) \leq(1+r) d(f z, g y) .
$$

Thus,

$$
\begin{aligned}
\varphi(r) d(g y, S y) & =(1-r) d(g y, S y) \\
& \leq\left(\frac{1}{1+r}\right) d(g y, S y) \leq d(f z, g y) .
\end{aligned}
$$

Then

$$
\varphi(r) \min \{d(f z, S z), d(g y, S y)\} \leq d(f z, g y),
$$

and by the assumption,

$$
\begin{aligned}
& H(S z, S y) \\
& \leq r \max \{d(f z, g y), d(f z, S z), d(g y, S y), \\
& \left.\frac{d(g y, S z)+d(f z, S y)}{2}\right\} .
\end{aligned}
$$

If $d(f z, g y)<d(g y, S y)$, then (38) gives

$$
d(g y, S y) \leq d(f z, g y)+r d(g y, S y)+\frac{1}{n} d(f z, g y)
$$

that is, $(1-r) d(g y, S y) \leq(1+1 / n) d(f z, g y)$.

Making $n \rightarrow \infty, \varphi(r) d(g y, S y) \leq d(f z, g y)$.

Then $\varphi(r) \min \{d(f z, S z), d(g y, S y)\} \leq d(f z, g y)$, and by the assumption, we get (43).

Since $d\left(S z, f x_{2 n+2}\right) \leq H\left(S z, S x_{2 n+1}\right)$, taking $y=x_{2 n+1}$ in (43) and passing to the limit, we obtain

$$
d(S z, f z) \leq r d(f z, S z) .
$$

This gives $f z \in S z$; that is, $z$ is a coincidence point of $f$ and $S$. Analogously, $g z \in S z$. Thus, (I) and (II) are completely proved.

Further, if $Y=X, f z$ is a fixed point of $f$, and $S$ and $f$ are IT-commuting at $z$, then $f S z \subseteq S f z$. Therefore, $f z \in S z$ implies $f f z \in f S z \subseteq S f z$, so $f z \in S f z$. This proves that $u=f z$ is a common fixed point of $f$ and $S$. This proves (III). Analogously, $S$ and $g$ have a common fixed point $g z_{1}$. Therefore (20) implies that $u$ is a common fixed point of $S$ and $g$. This proves (IV). Now (V) is immediate.

Remark 7. In Theorem 6, the hypothesis " $f z$ is a fixed point of $f$ " is essential for the existence of a common fixed point of $S$ and $f$ (see also [8]). Similarly, the hypothesis " $g z_{1}$ is a fixed point of $g$ " is essential for the existence of a common fixed point of $S$ and $g$. Further, the contractive condition for three maps $S: Y \rightarrow C L(X)$ and $f, g: Y \rightarrow X$ studied by Abbas et al. [23] are included in the assumptions of Theorem 6 .

Corollary 8. Theorem 2.

Proof. It comes from Theorem 6 when $g=f$. 
The following result due to Dorić and Lazović [25] generalizing many fixed point theorems is obtained as a special case from Theorem 6 when $Y=X$ and $f$ and $g$ are the identity map on $X$.

Corollary 9. Let $(X, d)$ be a complete metric space and $S$ : $X \rightarrow C L(X)$. Assume there exists $r \in[0,1)$ such that for every $x, y \in X$,

$$
\begin{array}{r}
\varphi(r) d(x, S x) \leq d(x, y) \\
\text { implies } H(S x, S y) \leq r M(S x, S y) .
\end{array}
$$

Then there exists an element $z \in X$ such that $z \in S z$.

The following result extends and generalizes coincidence and fixed point theorems of Fisher [37], Goebel [38], Jungck [17], and others.

Corollary 10. Let $f, g, P: Y \rightarrow X$ be such that $P(Y) \subseteq$ $f(Y) \cap g(Y)$. Let $P(Y)$ or $f(Y)$ or $g(Y)$ be a complete subspace of $X$. Assume there exists $r \in[0,1)$ such that for every $x, y \in Y$,

$$
\varphi(r) \min \{d(f x, P x), d(g y, P y)\} \leq d(f x, g y),
$$

implies

$$
d(P x, P y) \leq r M(P ; f x, g y) .
$$

Then $C(P, f)$ and $C(P, g)$ are nonempty. Further, if $Y=X$ and if $P$ commutes with $f$ and $g$ at a common coincidence point, then $f, g$, and $P$ have a unique common fixed point; that is, there exists a unique point $z \in X$ such that $f z=g z=P z=z$.

Proof. Set $S x=\{P x\}$ for every $x \in Y$. Then it easily comes from Theorem 6 that $C(P, f)$ and $C(P, g)$ are nonempty. Further, if $Y=X$ and $P$ commutes with $f$ and $g$ at $z$, then $f f z=f P z=P f z$ and $g g z=g P z=P g z$.

Also $\varphi(r) \min \{d(f z, P z), d(f f z, P f z)\}=0 \leq d(f z, f f z)$, and this implies

$$
\begin{aligned}
d(P z, P f z) \leq r \max & \{d(f z, f f z), d(f z, P z), d(f f z, P f z), \\
\left.\frac{d(f z, P f z)+d(f f z, P z)}{2}\right\} & \\
& =r d(P z, P f z) .
\end{aligned}
$$

This says that $f z$ is fixed point of $f$ and $P$. Analogously $g z$ is fixed point of $g$ and $P$. The uniqueness of the common fixed point follows easily.

Corollary 11. Let $(X, d)$ be a complete metric space and let $f, g: X \rightarrow X$ be an onto maps. Assume there exists $r \in[0,1)$ such that for every $x, y \in X$,

$$
\begin{array}{r}
\varphi(r) \min \{d(x, f x), d(y, g y)\} \leq d(f x, g y) \\
\text { implies } d(x, y) \leq r M_{1}(f x, g y) .
\end{array}
$$

Then $f$ and $g$ have a unique common fixed point.
Proof. It comes from Corollary 10 when $Y=X$ and $P$ is the identity map on $X$.

Corollary 12. Let $(X, d)$ be a complete metric space and let $f: X \rightarrow X$ be onto maps. Assume there exists $r \in[0,1)$ such that for every $x, y \in X$,

$$
\begin{array}{r}
\varphi(r) d(x, f x) \leq d(f x, f y) \\
\text { implies } d(x, y) \leq r M(f x, f y) .
\end{array}
$$

Then $f$ has a unique fixed point.

Proof. It comes from Corollary 11 when $f=g$.

The following example shows that Theorem 6 is indeed more general than Theorem 1.

Example 13. Consider a metric space $X=$ $\{(0,0),(0,1),(1,0),(1,2),(2,1)\}$, where $d$ is defined by

$$
d\left[\left(x_{1}, x_{2}\right),\left(y_{1}, y_{2}\right)\right]=\left|x_{1}-y_{1}\right|+\left|x_{2}-y_{2}\right| \text {. }
$$

Let $S, f$ and $g: X \rightarrow X$ be such that

$$
\begin{aligned}
& S\left(x_{1}, x_{2}\right)= \begin{cases}(0,0) & \text { if }\left(x_{1}, x_{2}\right) \neq(1,2),(2,1) \\
(0,1) & \text { if }\left(x_{1}, x_{2}\right)=(1,2) \\
(1,0) & \text { if }\left(x_{1}, x_{2}\right)=(2,1),\end{cases} \\
& f\left(x_{1}, x_{2}\right)=\left(x_{2}, x_{1}\right) \quad \forall\left(x_{1}, x_{2}\right) \in X, \\
& g\left(x_{1}, x_{2}\right)= \begin{cases}\left(x_{1}, x_{2}\right) & \text { if }\left(x_{1}, x_{2}\right) \neq(1,0) \\
(0,1) & \text { if }\left(x_{1}, x_{2}\right)=(1,0) .\end{cases}
\end{aligned}
$$

It is readily verified that

$$
\begin{gathered}
d(S x, S y) \\
\leq \frac{1}{2} \max \{d(f x, g y), d(f x, S x), d(g y, S y), \\
\left.\frac{d(S x, g y)+d(S y, f x)}{2}\right\},
\end{gathered}
$$

for all $(x, y) \in X$ except for $x, y \in\{(1,2),(2,1)\}$ with $r=1 / 2$.

For $x, y \in\{(1,2),(2,1)\}$, condition (3) yields $2 \leq 2 r$, which contradicts $0 \leq r<1$. Therefore, the condition (3) of Theorem 1 is not satisfied. So, in order to see that the maps $S, f$, and $g$ satisfy the assumption of Theorem 6 , we notice that the condition (9a) of Theorem 6 does not hold for $x, y \in\{(1,2),(2,1)\}$. Indeed, for $(x, y)=((1,2),(2,1))$,

$$
\begin{aligned}
\varphi( & (r) \min \{d(f x, S x), d(g y, T y)\} \\
& =\varphi(r) \min \{d(f(1,2), S(1,2)), d(g(2,1), T(2,1))\} \\
& =\varphi(r) \min \{2,2\}=2 \varphi(r) .
\end{aligned}
$$

That is, $\varphi(r) \min \{d(f x, S x), d(g y, T y)\}=1>0=d(f x, g y)$.

This violates (9a) when $\varphi(r)=1 / 2$ (as $r=1 / 2)$. Similarly (9a) is also not true for $(x, y)=((2,1),(1,2))$. It is easily seen that all other hypotheses of Theorem 6 are also true.

Now we give an application of Corollary 10. 
Theorem 14. Let $S: Y \rightarrow B N(X)$ and $f, g: Y \rightarrow X$ be such that $S(Y) \subseteq f(Y) \cap g(Y)$, and let one of $S(Y), f(Y)$, or $g(Y)$ be a complete subspace of $X$. Assume there exists $r \in[0,1)$ such that for every $x, y \in Y$,

$$
\varphi(r) \min \{\rho(f x, S x), \rho(g y, S y)\} \leq d(f x, g y),
$$

implies

$$
\begin{gathered}
\rho(S x, S y) \\
\leq r \max \{d(f x, g y), \rho(f x, S x), \rho(g y, S y), \\
\left.\frac{d(f x, S y)+d(g y, S x)}{2}\right\} .
\end{gathered}
$$

Then $C(S, f)$ and $C(S, g)$ are nonempty.

Proof. Choose $\lambda \in(0,1)$. Define single-valued maps $h_{1}, h_{2}$ : $X \rightarrow X$ as follows. For each $x \in X$, let $h_{1} x$ be a point of $S x$ which satisfies

$$
d\left(f x, h_{1} x\right) \geq r^{\lambda} \rho(f x, S x)
$$

Similarly, for each $y \in X$, let $h_{2} y$ be a point of $S y$ such that

$$
d\left(g y, h_{2} y\right) \geq r^{\lambda} \rho(g y, S y)
$$

Since $h_{1} x \in S x$ and $h_{2} y \in S y$,

$$
d\left(f x, h_{1} x\right) \leq \rho(f x, S x), \quad d\left(g y, h_{2} y\right) \leq \rho(g y, S y) .
$$

So (56) gives

$$
\begin{aligned}
& \varphi(r) \min \left\{d\left(f x, h_{1} x\right), d\left(g y, h_{2} y\right)\right\} \\
& \quad \leq \varphi(r) \min \{\rho(f x, S x), \rho(g y, S y)\} \leq d(f x, g y),
\end{aligned}
$$

and this implies (57). Therefore,

$$
\begin{aligned}
& d\left(h_{1} x, h_{2} y\right) \\
& \leq \rho(S x, S y) \\
& \leq r \cdot r^{-\lambda} \max \left\{r^{\lambda} d(f x, g y), r^{\lambda} \rho(f x, S x), r^{\lambda} \rho(g y, S y),\right. \\
& \leq r^{1-\lambda} \max \left\{d(f x, g y), d\left(f x, h_{1} x\right), d\left(g y, h_{2} y\right),\right. \\
& \left.\frac{d\left(f x, h_{2} y\right)+d\left(g y, h_{1} x\right)}{2}\right\} .
\end{aligned}
$$

So (61), namely, $\varphi\left(r^{\prime}\right) \min \left\{d\left(f x, h_{1} x\right), d\left(g y, h_{2} y\right)\right\} \leq d(f x$, gy), implies

$$
\begin{gathered}
d\left(h_{1} x, h_{2} y\right) \leq r^{\prime} \max \left\{d(f x, g y), d\left(f x, h_{1} x\right), d\left(g y, h_{2} y\right),\right. \\
\left.\frac{d\left(f x, h_{2} y\right)+d\left(g y, h_{1} x\right)}{2}\right\},
\end{gathered}
$$

where $r^{\prime}=r^{1-\lambda}<1$.

Hence, by Corollary 10, there exist $z_{1}, z_{2} \in Y$ such that $h_{1} z_{1}=f z_{1}$ and $h_{2} z_{2}=g z_{2}$. This implies that $z_{1}$ is a coincidence point of $f$ and $S$, and $z_{2}$ is a coincidence point of $g$ and $S$.

Corollary 15. Let $S: Y \rightarrow B N(X)$ and $f: Y \rightarrow X$ be such that $S(Y) \subseteq f(Y)$, and let $S(Y)$ or $f(Y)$ be a complete subspace of $X$. Assume there exists $r \in[0,1)$ such that for every $x, y \in Y$,

$$
\varphi(r) \rho(f x, S x) \leq d(f x, f y)
$$

implies

$$
\begin{gathered}
\rho(S x, S y) \\
\leq r \max \{d(f x, f y), \rho(f x, S x), \rho(f y, S y), \\
\left.\frac{d(f x, S y)+d(f y, S x)}{2}\right\} .
\end{gathered}
$$

Then there exists $z \in Y$ such that $f z \in S z$.

Proof. It comes from Theorem 14 when $g=f$.

Corollary 16. Let $X$ be a complete metric space and let $S$ : $X \rightarrow B N(X)$. Assume there exists $r \in[0,1)$ such that for every $x, y \in X$,

$$
\varphi(r) \rho(x, S x) \leq d(x, y)
$$

implies

$$
\begin{gathered}
\rho(S x, S y) \\
\leq r \max \{d(x, y), \rho(x, S x), \rho(y, S y), \\
\left.\frac{d(x, S y)+d(y, S x)}{2}\right\} .
\end{gathered}
$$

Then there exists a unique point $z \in X$ such that $z \in S z$.

Proof. It comes from Theorem 14 that $S$ has a fixed point when $f=g$ is the identity map on $X$. The uniqueness of the fixed point follows easily.

\section{Applications}

Throughout this section, we assume that $U$ and $V$ are Banach spaces, $W \subseteq U$, and $D \subseteq V$. Let $R$ denote the field of reals, 
$\tau: W \times D \rightarrow W, g, g^{\prime}: W \times D \rightarrow R$, and $G, F_{1}, F_{2}: W \times$ $D \times R \rightarrow R$. Considering $W$ and $D$ as the state and decision spaces, respectively, the problem of dynamic programming reduces to the problem of solving the functional equations:

$$
\begin{gathered}
p=\sup _{y \in D}\{g(x, y)+G(x, y, p(\tau(x, y)))\}, \quad x \in W, \\
q_{i}=\sup _{y \in D}\left\{g^{\prime}(x, y)+F_{i}(x, y, q(\tau(x, y)))\right\}, \\
x \in W, \quad i=1,2 .
\end{gathered}
$$

Indeed, in the multistage process, some functional equations arise in a natural way (cf. Bellman [40] and Bellman and Lee [41]; see also [6, 9, 15, 28, 33, 42-45]). In this section, we study the existence of a common solution of the functional equations (68a) and (68b) arising in the dynamic programming.

Let $B(W)$ denote the set of all bounded real-valued functions on $W$. For an arbitrary $h \in B(W)$, define $\|h\|=$ $\sup _{x \in W}|h(x)|$. Then $(B(W),\|\cdot\|)$ is a Banach space. Suppose that the following conditions hold:

(DP-1) $G, F_{1}, F_{2}, g$, and $g$ are bounded.

(DP-2) Let $\varphi(r)$ be considered as in the previous sections. Assume that there exists $r \in[0,1)$ such that for every $(x, y) \in$ $W \times D, h, k \in B(W)$ and $t \in W$,

$$
\begin{aligned}
& \varphi(r) \min \left\{\left|J_{1} h(t)-A h(t)\right|,\left|J_{2} k(t)-A k(t)\right|\right\} \\
& \leq\left|J_{1} h(t)-J_{2} k(t)\right|,
\end{aligned}
$$

implies

$$
|G(x, y, h(t))-G(x, y, k(t))| \leq r M\left(A ; J_{1} h, J_{2} k\right),
$$

where

$$
\begin{aligned}
& M\left(A ; J_{1} h, J_{2} k\right) \\
&=\max \left\{\left|J_{1} h(t)-J_{2} k(t)\right|,\left|J_{1} h(t)-A h(t)\right|,\right. \\
& \\
& \quad\left|J_{2} k(t)-A k(t)\right|, \\
&\left.\frac{\left|J_{1} h(t)-A k(t)\right|+\left|J_{2} k(t)-A h(t)\right|}{2}\right\},
\end{aligned}
$$

and $A, J_{1}$, and $J_{2}$ are defined as follows:

$$
\begin{gathered}
A h(x)=\sup _{y \in D}\{g(x, y)+G(x, y, h(\tau(x, y)))\}, \\
x \in W, \quad h \in B(W), \\
J_{i} h(x)=q_{i}=\sup _{y \in D}\left\{g^{\prime}(x, y)+F_{i}(x, y, h(\tau(x, y)))\right\}, \\
x \in W, \quad h \in B(W), \quad i=1,2 .
\end{gathered}
$$
that

(DP-3) For any $h, k \in B(W)$, there exists $u, v \in B(W)$ such

$$
A h(x)=J_{1} u(x), \quad A k(x)=J_{2} v(x), \quad x \in W .
$$

(DP-4) There exists $h, k \in B(W)$ such that

$$
\begin{aligned}
& J_{1} h(x)=A h(x) \quad \text { implies } J_{1} A h(x)=A J_{1} h(x), \\
& J_{2} k(x)=A k(x) \quad \text { implies } J_{2} A k(x)=A J_{2} k(x) .
\end{aligned}
$$

Theorem 17. Assume the conditions (DP-1)-(DP-4). Let $J(B(W))$ be a closed convex subspace of $B(W)$. Then the functional equations (68a) and (68b), $i=1,2$, have a unique bounded common solution in $B(W)$.

Proof. For any $h, k \in B(W)$, let $d(h, k)=\sup \{|h(x)-k(x)|$ : $x \in W\}$. Then $(B(W), d)$ is a complete metric space. By virtue of (DP-3) and (DP-4), $A(B(W)) \subseteq J_{1}(B(W)) \cap J_{2}(B(W))$ and the map $A$ is IT-commuting with $J_{1}$ and $J_{2}$ at coincidence points.

Let $\lambda$ be an arbitrary positive number and $h_{1}, h_{2} \in B(W)$. Pick $x \in W$, and choose $y_{1}, y_{2} \in D$ such that

$$
A h_{j}<g\left(x, y_{j}\right)+G\left(x, y_{j}, h_{j}\left(x_{j}\right)\right)+\lambda, \quad j=1,2,
$$

where $x_{j}=\tau\left(x, y_{j}\right)$. Further,

$$
\begin{aligned}
& A h_{1} \geq g\left(x, y_{2}\right)+G\left(x, y_{2}, h_{1}\left(x_{2}\right)\right), \\
& A h_{2} \geq g\left(x, y_{1}\right)+G\left(x, y_{1}, h_{2}\left(x_{1}\right)\right) .
\end{aligned}
$$

Therefore, the first inequality in (DP-2) becomes

$$
\begin{aligned}
& \varphi(r) \min \left\{\left|J_{1} h_{1}(x)-A h_{1}(x)\right|,\left|J_{2} h_{2}(x)-A h_{2}(x)\right|\right\} \\
& \leq\left|J_{1} h_{1}(x)-J_{2} h_{2}(x)\right|,
\end{aligned}
$$

and this together with (75), (77), and (78) implies

$$
\begin{aligned}
A h_{1}-A h_{2} & <G\left(x, y_{1}, h_{1}\left(x_{1}\right)\right)-G\left(x, y_{1}, h_{2}\left(x_{1}\right)\right)+\lambda \\
& \leq\left|G\left(x, y_{1}, h_{1}\left(x_{1}\right)\right)-G\left(x, y_{1}, h_{2}\left(x_{1}\right)\right)\right|+\lambda \\
& \leq r M\left(A ; J_{1} h_{1}, J_{2} h_{2}\right)+\lambda .
\end{aligned}
$$

Similarly, (75), (76), and (78) imply

$$
A h_{2}(x)-A h_{1}(x) \leq r M\left(A ; J_{1} h_{1}, J_{2} h_{2}\right)+\lambda .
$$

So, from (79) and (80), we obtain

$$
\left|A h_{1}(x)-A h_{2}(x)\right| \leq r M\left(A ; J_{1} h_{1}, J_{2} h_{2}\right)+\lambda .
$$

As $\lambda>0$ is arbitrary and (81) is true for any $x \in W$, taking supremum, we find from (78) and (81) that

$$
\varphi(r) \min \left\{d\left(J_{1} h_{1}, A h_{1}\right), d\left(J_{2} h_{2}, A h_{2}\right)\right\} \leq d\left(J_{1} h_{1}, J_{2} h_{2}\right),
$$

implies

$$
d\left(A h_{1}, A h_{2}\right) \leq r M\left(A ; J_{1} h_{1}, J_{2} h_{2}\right) .
$$

Therefore, Corollary 10 applies, wherein $A, J_{1}$ and $J_{2}$ correspond, respectively, to the maps $P, f$, and $g$. So $\left(A, J_{1}\right)$ and $\left(A, J_{2}\right)$ have a unique common fixed point $h^{*}$; that is, $h^{*}(x)$ is the unique bounded common solution of the functional equations (68a) and (68b), $i=1,2$.

Now we furnish an example in support of Theorem 17. 
Example 18. Let $X=Y=R$ be a Banach space endowed with the standard norm $\|\cdot\|$ defined by $\|x\|=|x|$, for all $x \in X$.

Suppose $W=[0,1] \subseteq X$ be the state space and $D=$ $[0, \infty) \subseteq Y$ the decision space. Define $\tau: W \times D \rightarrow W$ by

$$
\tau(x, y)=\frac{x}{y^{2}+1}, \quad x \in W, y \in D
$$

For any $h, k \in B(W)$ and $i=1,2$, define $p, q_{i}: W \rightarrow R$ by

$$
p(x)=q_{i}(x)=x^{2}+\frac{1}{2} .
$$

Define $G, F_{1}, F_{2}, g, g^{\prime}: W \times D \times R \rightarrow R$ by

$$
\begin{aligned}
G(x, y, t) & =\frac{1}{4}\left\{\frac{x}{(x+1)(y+1)} \sin \frac{y}{y+1}+2\right\}, \\
F_{1}(x, y, t) & =\frac{1}{2 x+y+1}+\frac{1}{2} \sin t \\
F_{2}(x, y, t) & =\frac{1}{2 x+3 y+1}+\frac{1}{2} \sin t, \\
g(x, y) & =\frac{x^{2} y^{2}}{x+y^{2}}, \quad g^{\prime}(x, y)=\frac{x^{2} y^{5}}{x+y^{5}} .
\end{aligned}
$$

Notice that $G, F_{1}, F_{2}, g$, and $g^{\prime}$ are bounded. Also

$$
\begin{aligned}
J_{1} h(x)= & \sup _{y \in D}\left\{g^{\prime}(x, y)+F_{1}(x, y, h(\tau(x, y)))\right\}, \\
= & x^{2}+\frac{1}{2}=q_{1}(x) ; \\
& x \in W, \quad h \in B(W), \\
J_{2} k(x)= & \sup _{y \in D}\left\{g^{\prime}(x, y)+F_{2}(x, y, k(\tau(x, y)))\right\}, \\
= & x^{2}+\frac{1}{2}=q_{2}(x) ; \\
A h(x)= & \sup _{y \in D}\{g(x, y)+G(x, y, h(\tau(x, y)))\}, \\
= & x^{2}+\frac{1}{2}=p(x) ; \\
& \quad x \in W, \quad h \in B(W), \\
A k(x)= & \sup _{y \in D}\{g(x, y)+G(x, y, k(\tau(x, y)))\}, \\
= & x^{2}+\frac{1}{2}=p(x) ;
\end{aligned}
$$

Now

$$
\begin{aligned}
& \varphi(r) \min \left\{\left|J_{1} h(t)-A h(t)\right|,\left|J_{2} k(t)-A k(t)\right|\right\} \\
& \quad=\varphi(r) \min \left\{\left|q_{1}(x)-p(x)\right|,\left|q_{2}(x)-p(x)\right|\right\} \\
& \quad=0=\left|J_{1} h(t)-J_{2} k(t)\right| .
\end{aligned}
$$

Thus,

$$
\begin{gathered}
\varphi(r) \min \{|J h(t)-A h(t)|,|J k(t)-A k(t)|\} \\
=|J h(t)-J k(t)|,
\end{gathered}
$$

and this implies

$$
|G(x, y, h(t))-G(x, y, k(t))|=0 \leq r M(A ; J h(t), J k(t)) .
$$

Finally, for any $h, k \in B(W)$ with $A h=J h$, we have

$$
A J h=p(x)=q(x)=J J h=J A h ;
$$

that is, $J A h=A J h$, and with $A k=J k$, we have $A J k=p(x)=$ $q(x)=J J k=J A k$; that is, $J A k=A J k$.

Thus, all the hypotheses of Theorem 17 are satisfied. So the system of (68a) and (68b) has a unique solution in $B(W)$.

Corollary 19. Suppose that the following conditions hold.

(i) $G, F, g$, and $g^{\prime}$ are bounded.

(ii) Assume there exists $r \in[0,1)$ such that for every $(x, y) \in W \times D, h, k \in B(W)$ and $t \in W$,

$$
\varphi(r)|J h(t)-A h(t)| \leq|J h(t)-J k(t)|,
$$

implies

$$
\begin{aligned}
\mid G(x, y, h(t))- & G(x, y, k(t)) \mid \\
\leq r \max \{|J h(t)-J k(t)|,|J h(t)-A h(t)|, & \\
& |J k(t)-A k(t)|, \\
& \left.\frac{|J h(t)-A k(t)|+|J k(t)-A h(t)|}{2}\right\},
\end{aligned}
$$

where $A$ and $J$ are defined as follows:

$$
\begin{array}{r}
A h(x)=\sup _{y \in D}\{g(x, y)+G(x, y, h(\tau(x, y)))\}, \\
x \in W, \quad h \in B(W), \\
J h(x)=q=\sup _{y \in D}\left\{g^{\prime}(x, y)+F(x, y, h(\tau(x, y)))\right\}, \\
x \in W, \quad h \in B(W) .
\end{array}
$$
that

(iii) For any $h, k \in B(W)$, there exists $u, v \in B(W)$ such

$$
A h(x)=J u(x), \quad A k(x)=J v(x), \quad x \in W .
$$


(iv) There exists $h, k \in B(W)$ such that

$$
\begin{aligned}
& J h(x)=A h(x) \quad \text { implies } J A h(x)=A J h(x), \\
& J k(x)=A k(x) \quad \text { implies JAk }(x)=A J k(x) .
\end{aligned}
$$

Then the functional equations (68a) and (68b) with $F_{1}=F_{2}=$ $F$ possess a unique bounded common solution in $W$.

Proof. It comes from Theorem 17 when $F_{1}=F_{2}=F$.

Now we derive the the following result due to Dorić and Lazović [25], which in turn extends certain results from [41, 42].

Corollary 20. Suppose that the following conditions hold.

(i) $G$ and $g$ are bounded.

(ii) There exists $r \in[0,1)$ such that for every $(x, y) \in W \times$ $D, h, k \in B(W)$ and $t \in W$,

$$
\varphi(r)|h(t)-K h(t)| \leq|h(t)-k(t)|
$$

implies

$$
\begin{aligned}
& |G(x, y, h(t))-G(x, y, k(t))| \\
& \quad \leq r \max M(K, K ; h(t), k(t)),
\end{aligned}
$$

where $K$ is defined as

$$
\begin{array}{r}
A h(x)=\sup _{y \in D}\{g(x, y)+G(x, y, h(\tau(x, y)))\}, \\
x \in W, \quad h \in B(W) .
\end{array}
$$

Then the functional equation (68a) with $G_{1}=G_{2}=$ G possesses a unique bounded solution in $W$.

Proof. It comes from Corollary 19 when $g^{\prime}=0, \tau(x, y)=$ $x$ and $F(x ; y ; t)=t$ as the assumption (DP-3) becomes redundant in this context.

\section{Conflict of Interests}

The authors declare that there is no conflict of interests regarding the publication of this paper.

\section{Acknowledgments}

The authors thank the referees for their appreciation and suggestions to improve upon the original typescript. The authors are grateful to the Spanish Government for its support of this research through Grant DPI2012-30651 and to the Basque Government for its support of this research through Grants IT378-10 and SAIOTEK S-PE12UN015. They are also grateful to the University of Basque Country for its financial support through Grant UFI 2011/07.

\section{References}

[1] S. B. Nadler, Jr., "Multi-valued contraction mappings," Pacific Journal of Mathematics, vol. 30, pp. 475-488, 1969.
[2] M. A. Khamsi and W. A. Kirk, An introduction to metric spaces and fixed point theory, Wiley-Interscience, New York, NY, USA, 2001.

[3] I. A. Rus, Generalized Contractions and Applications, Cluj University Press, Cluj-Napoca, Romania, 2001.

[4] R. P. Agarwal, M. Meehan, and D. O’Regan, Fixed Point Theory and Applications, vol. 141, Cambridge University Press, Cambridge, UK, 2001.

[5] L. B. Ćirić, "Fixed points for generalized multi-valued contractions," Matematički Vesnik, vol. 9, no. 24, pp. 265-272, 1972.

[6] S. Czerwik, Fixed Point Theorems and Special Solutions of Functional Equations, vol. 428, Uniwersytetslqski, Katowice, Poland, 1980.

[7] O. Hadžić, "A coincidence theorem for multivalued mappings in metric spaces," Universitatas Babeş-Bolyai. Studia. Mathematica, vol. 26, no. 4, pp. 65-67, 1981.

[8] S. A. Naimpally, S. L. Singh, and J. H. M. Whitfield, "Coincidence theorems for hybrid contractions," Mathematische Nachrichten, vol. 127, pp. 177-180, 1986.

[9] H. K. Pathak, Y. J. Cho, S. M. Kang, and B. S. Lee, "Fixed point theorems for compatible mappings of type $(P)$ and applications to dynamic programming," Le Matematiche, vol. 50, no. 1, pp. 15-33, 1995.

[10] A. Petrusel and I. A. Rus, "The theory of a metric fixed point theorem for multivalued operators," in Proceedings of the 9th International Conference on Fixed Point Theory and Its Applications, pp. 161-175, Yokohama, Changhua, Taiwan, July 2009.

[11] S. Reich, "Fixed points of multi-valued functions," Atti della Accademia Nazionale dei Lincei. Rendiconti. Classe di Scienze Fisiche, Matematiche e Naturali, vol. 8, no. 51, pp. 32-35, 1971.

[12] S. L. Singh and S. N. Mishra, "Nonlinear hybrid contractions," Journal of Natural \& Physical Sciences, vol. 5-8, pp. 191-206, 1991/94.

[13] S. L. Singh and S. N. Mishra, "On general hybrid contractions," Australian Mathematical Society A, vol. 66, no. 2, pp. 244-254, 1999.

[14] R. Wegrzyk, "Fixed-point theorems for multivalued functions and their applications to functional equations," Polska Akademia Nauk. Instytut Matematyczny. Dissertationes Mathematicae. Rozprawy Matematyczne, vol. 201, pp. 1-28, 1982.

[15] H. K. Pathak, "Fixed point theorems for weak compatible multi-valued and single-valued mappings," Acta Mathematica Hungarica, vol. 67, no. 1-2, pp. 69-78, 1995.

[16] H. Covitz and S. B. Nadler, Jr., "Multi-valued contraction mappings in generalized metric spaces," Israel Journal of Mathematics, vol. 8, pp. 5-11, 1970.

[17] G. Jungck, "Commuting mappings and fixed points," The American Mathematical Monthly, vol. 83, no. 4, pp. 261-263, 1976.

[18] R. Kamal, R. Chugh, S. L. Singh, and S. N. Mishra, "New common fixed point theorems for multivalued maps," Applied General Topology, 2013.

[19] S. Itoh and W. Takahashi, "Single-valued mappings, multivalued mappings and fixed-point theorems," Journal of Mathematical Analysis and Applications, vol. 59, no. 3, pp. 514-521, 1977.

[20] G. Jungck and B. E. Rhoades, "Fixed points for set valued functions without continuity," Indian Journal of Pure and Applied Mathematics, vol. 29, no. 3, pp. 227-238, 1998.

[21] J. Jachymski, "Common fixed point theorems for some families of maps," Indian Journal of Pure and Applied Mathematics, vol. 25, no. 9, pp. 925-937, 1994. 
[22] S. B. Nadler, Jr., Hyperspaces of Sets, Marcel Dekker, New York, NY, USA, 1978.

[23] M. Abbas, B. Ali, and S. N. Mishra, "Fixed points of multivalued Suzuki-Zamfirescu- $(f, g)$ contraction mappings," Matematički Vesnik, vol. 66, no. 1, pp. 58-72, 2014.

[24] S. Dhompongsa and H. Yingtaweesittikul, "Fixed points for multivalued mappings and the metric completeness," Fixed Point Theory and Applications, Article ID 972395, 15 pages, 2009.

[25] D. Dorić and R. Lazović, "Some Suzuki-type fixed point theorems for generalized multivalued mappings and applications," Fixed Point Theory and Applications, vol. 2011, article 40, 2011.

[26] G. Moț and A. Petruşel, "Fixed point theory for a new type of contractive multivalued operators," Nonlinear Analysis, vol. 70, no. 9, pp. 3371-3377, 2009.

[27] S. L. Singh and S. N. Mishra, "Coincidence theorems for certain classes of hybrid contractions," Fixed Point Theory and Applications, vol. 2010, Article ID 898109, 14 pages, 2010.

[28] S. L. Singh, S. N. Mishra, R. Chugh, and R. Kamal, "General common fixed point theorems and applications," Journal of Applied Mathematics, vol. 2012, Article ID 902312, 14 pages, 2012.

[29] T. Kubiak, "Two coincidence theorems for contractive type multivalued mappings," Universitatis Babeş-Bolyai. Studia. Mathematica, vol. 30, pp. 65-68, 1985.

[30] M. Kikkawa and T. Suzuki, "Three fixed point theorems for generalized contractions with constants in complete metric spaces," Nonlinear Analysis, vol. 69, no. 9, pp. 2942-2949, 2008.

[31] S. L. Singh and S. N. Mishra, "Fixed point theorems for singlevalued and multi-valued maps," Nonlinear Analysis, vol. 74, no. 6, pp. 2243-2248, 2011.

[32] S. L. Singh, R. Chugh, and R. Kamal, "Suzuki type common fixed point theorems and applications," Fixed Point Theory, vol. 14, no. 2, pp. 497-505, 2013.

[33] S. L. Singh, R. Chugh, and R. Kamal, "Suzuki type hybrid contractions and applications," Indian Journal of Mathematics, vol. 56, no. 1, pp. 49-76, 2014.

[34] S. L. Singh and A. M. Hashim, "New coincidence and fixed point theorems for strictly contractive hybrid maps," The Australian Journal of Mathematical Analysis and Applications, vol. 2, no. 1, pp. 1-7, 2005.

[35] B. Djafari Rouhani and S. Moradi, "Common fixed point of multivalued generalized $\varphi$-weak contractive mappings," Fixed Point Theory and Applications, Article ID 708984, 13 pages, 2010.

[36] S. L. Singh and S. N. Mishra, "Remarks on recent fixed point theorems," Fixed Point Theory and Applications, Article ID 452905, 18 pages, 2010.

[37] B. Fisher, "Mappings with a common fixed point," Kobe University. Mathematics Seminar Notes, vol. 7, no. 1, pp. 81-84, 1979.

[38] K. Goebel, "A coincidence theorem," Bulletin de l'Académie Polonaise des Sciences. Série des Sciences Mathématiques, vol. 16, pp. 733-735, 1968.

[39] T. Suzuki, "A generalized Banach contraction principle that characterizes metric completeness," Proceedings of the American Mathematical Society, vol. 136, no. 5, pp. 1861-1869, 2008.

[40] R. Bellman, Methods of Nonliner Analysis, vol. 2, Academic Press, New York, NY, USA, 1973.

[41] R. Bellman and E. S. Lee, "Functional equations in dynamic programming," Aequationes Mathematicae, vol. 17, no. 1, pp. 118,1978 .
[42] P. C. Bhakta and S. Mitra, "Some existence theorems for functional equations arising in dynamic programming," Journal of Mathematical Analysis and Applications, vol. 98, no. 2, pp. 348-362, 1984.

[43] H. K. Pathak and Deepmala, "Some existence theorems for solvability of certain functional equations arising in dynamic programming," Bulletin of the Calcutta Mathematical Society, vol. 104, no. 3, pp. 237-244, 2012.

[44] H. K. Pathak and R. Tiwari, "Common fixed points for weakly compatible mappings and applications in dynamic programming," Italian Journal of Pure and Applied Mathematics, no. 30, pp. 253-268, 2013.

[45] S. L. Singh and S. N. Mishra, "On a Ljubomir Ćirić fixed point theorem for nonexpansive type maps with applications," Indian Journal of Pure and Applied Mathematics, vol. 33, no. 4, pp. 531$542,2002$. 


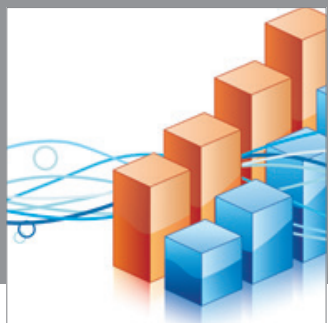

Advances in

Operations Research

mansans

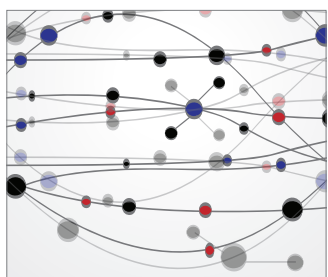

The Scientific World Journal
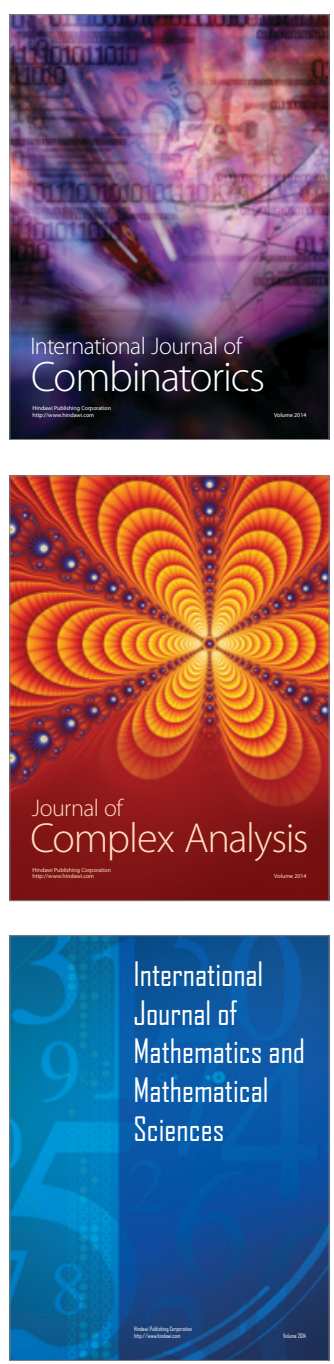
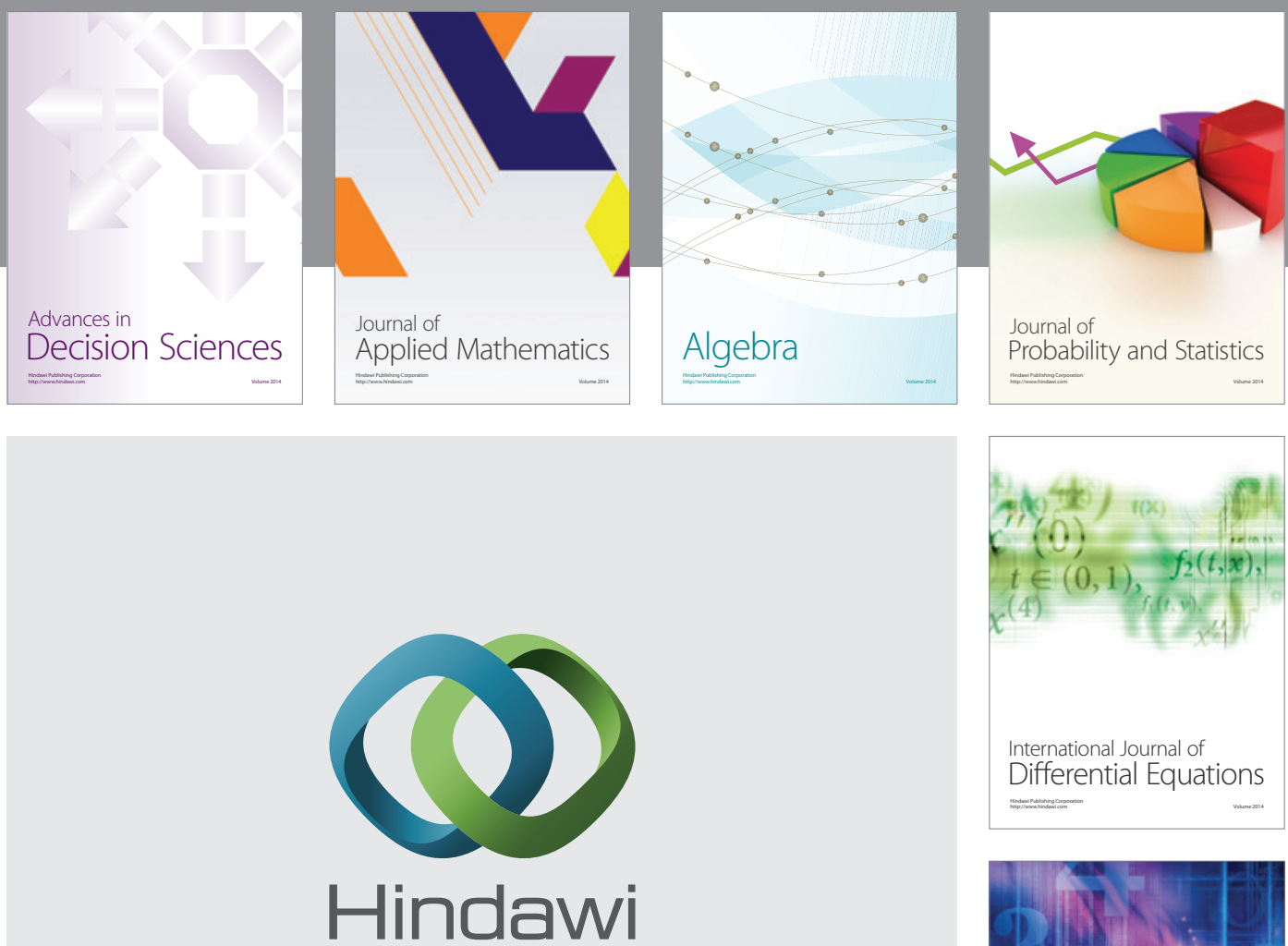

Submit your manuscripts at http://www.hindawi.com
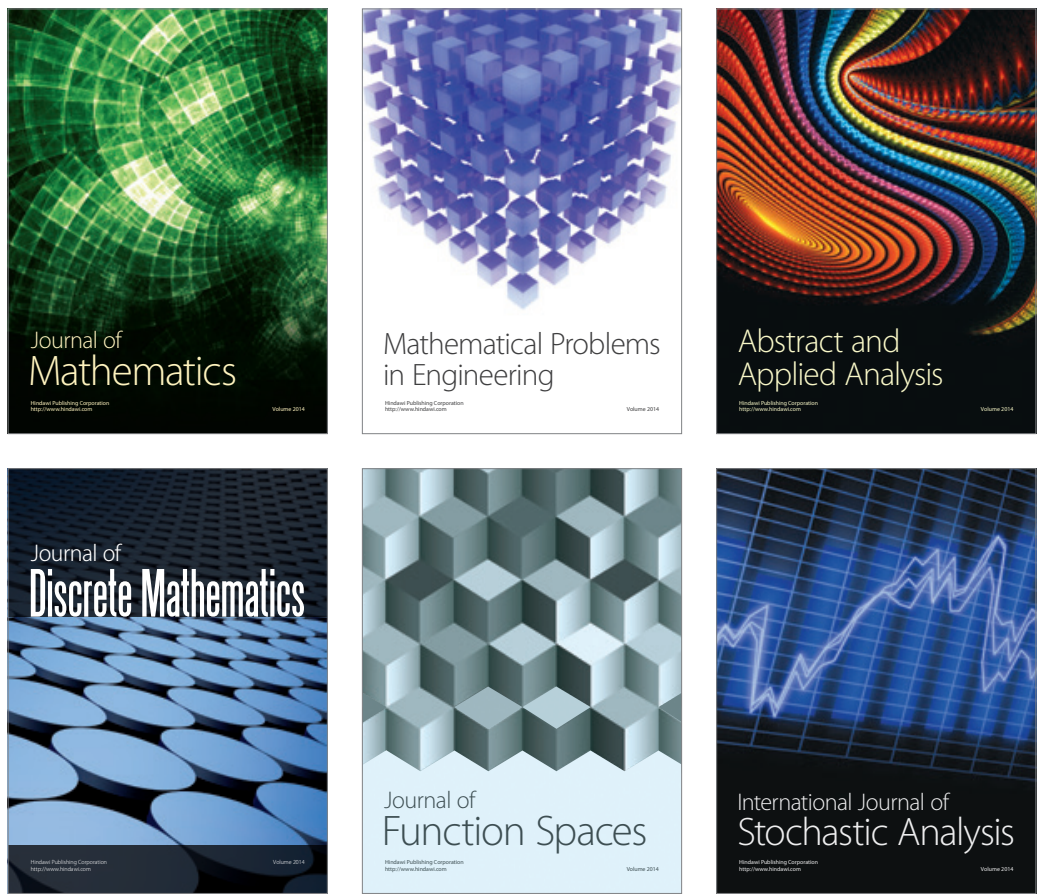

Journal of

Function Spaces

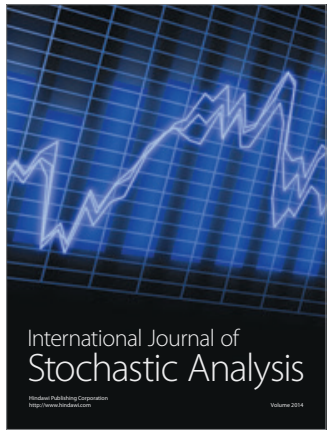

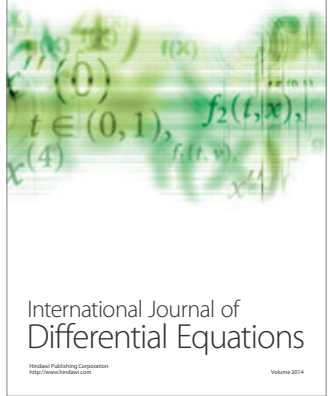
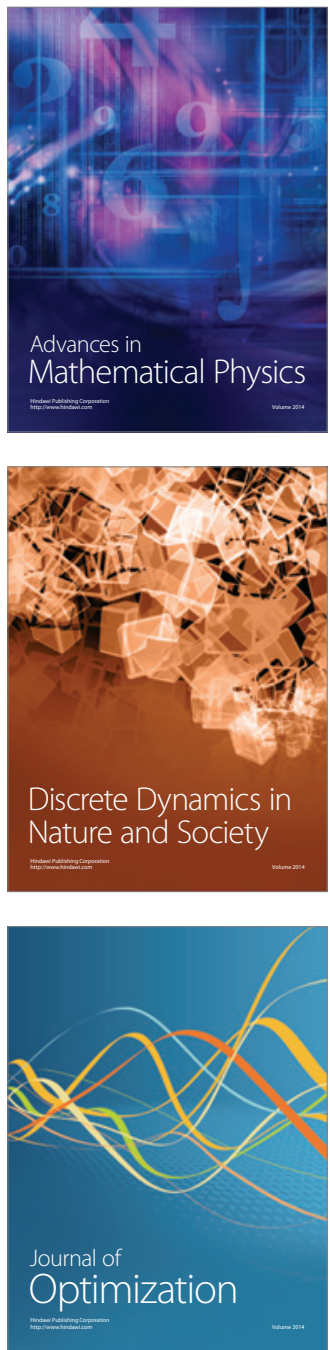